Voix et Images

\title{
Notion et/ou fonctions de la littérature (nationale québécoise) au $\mathrm{XX}^{\mathrm{e}}$ siècle
}

\section{Robert Giroux}

Volume 5, numéro 1, automne 1979

Jacques Godbout

URI : https://id.erudit.org/iderudit/200190ar

DOI : https://doi.org/10.7202/200190ar

Aller au sommaire du numéro

Éditeur(s)

Les Presses de l'Université du Québec

\section{ISSN}

0318-9201 (imprimé)

1705-933X (numérique)

Découvrir la revue

Citer cet article

Giroux, R. (1979). Notion et/ou fonctions de la littérature (nationale québécoise) au XX ${ }^{\mathrm{e}}$ siècle. Voix et Images, 5(1), 87-116. https://doi.org/10.7202/200190ar d'utilisation que vous pouvez consulter en ligne.

https://apropos.erudit.org/fr/usagers/politique-dutilisation/ 


\title{
Notion et/ou fonctions de la littérature (nationale québécoise) au $\mathrm{XX}{ }^{e}$ siècle
}

\begin{abstract}
* En somme quand on parle littérature [...] on est conduit soit à accepter sans discussion un donné préexistant - système de valeurs ou anthologie, - soit à s'en remettre à un jugement subjectif impressionniste, soit à tenter une rationalisation extérieure à la littérature elle-même, qui dissout les frontières du littéraire et du non-littéraire et fait disparaître l'objet même qu'on prétend étudier.

II en résulte que si ce que nous appelons "littérature" au XXं $\mathrm{XX}^{\ominus}$ siecle est l'institution qui permet à la société d'imposer ses structures à l'au-delà du langage, toute manifestation d'une littérature vivante caractérisée par la liberté d'écriture de l'écrivain est, de nos jours, à quelque degré antilittérature. Autrement dit la littérature comme fait historique concret [...] porte en elle sa propre négation et aboutit à son propre dépassement. $n 1$
\end{abstract}

Nous sommes plus que jamais dans l'ère du soupçon de la littérature. Et c'est la raison pour laquelle nous avons cru bon de citer longuement Escarpit dont les réflexions sont encore bien d'actualité et insuffisamment connues. La sophistication des approches (lectures) critiques universitaires n'a réussi jusqu'ici qu'à retarder une réflexion en profondeur de ce qu'est le phénomène littéraire. Le mot littérature n'existe d'ailleurs que depuis deux siècles et l'expression consacrée, du moins en occident, de faire de la littérature, d'étudier un champ textuel sur la valeur duquel on n'a pas à s'interroger, en dit long sur l'unanimité du discours littéraire qu'on nous propose comme universellement valide. Ce «on impersonnel demande à être circonscrit; il n'est pas neutre, loin de là. Qui écrit et pourquoi, pour qui ? Qui lit et pourquoi certains textes sont-ils retenus comme littéraires tandis que d'autres sont 
relégués aux oubliettes ou parfois ou purgatoire, duquel ils ressortent transformés par on ne sait quel pouvoir occulte?

Depuis toujours, parler de la littérature a été une pratique abandonnée à la critique, doublant ainsi la pratique de l'écriture, permettant à un écrit de devenir texte, œuvre, etc. La littérature n'a pourtant jamais été un corpus stable de chefs-d'œuvres. L'étude de la genèse des textes n'a jamais suffi à expliquer sérieusement la production de tel type de textes à telle époque. Le texte littéraire ne peut pas non plus valoir uniquement par des traits qui lui seraient spécifiques, immanents, distincts de traits qui seraient non littéraires. Cette perspective ne permet que d'ébaucher une théorie des genres littéraires fondée sur des critères internes, une théorie des normes, variables d'une époque à l'autre. D'ailleurs comment expliquer que des textes aussi différents que les Essais de Montaigne, les Lettres persanes de Montesquieu, l'Émile de Rousseau, A la recherche du temps perdu de Proust, les textes divers de Sartre, etc, soient passés dans l'histoire de la littérature française? On reconnaît le même type de phénomène au Québec. C'est donc au niveau de sa consommation seule que se décide l'appartenance ou non d'un texte à la littérature, la classification de cette dernière et son fonctionnement dans une société, dans une culture, système de valeurs idéologiques, internes et externes au texte. Comment s'articulent les rapports entre écriture, littérature et idéologie?

Avant de nous aventurer au cœur des problèmes complexes què soulèvent toutes ces questions - préférant y revenir à la fin de notre étude, nous tenterons de dégager, à partir de deux moments historiques de l'évolution culturelle du Québec, quelle conception on se faisait de la pratique et de l'usage de la littérature. Le premier moment historique couvre l'entre-deux-guerres et le second le début des années '70. Entre ces deux dates, nous n'ignorons pas qu'à chaque décennie correspond une redéfinition du phénomène littéraire. Mais comme nous poursuivons moins un travail d'historien qu'un projet de théorie de l'histoire de la littérature, notre étude ne tend pas à brosser un tableau exhaustif des divers "mouvements " littéraires au Québec; elle cherche surtout à répondre à des hypothèses théoriques que des exemples puisés dans des moments historiques éloignés viendront confirmer ou infirmer.

Il s'agit de deux périodes littéraires coiffées respectivement d'une enquête réalisée sous forme d'interviews. La première enquête date de 1939 ; elle est due à Adrienne Choquette et constitue les Confidences d'écrivains canadiens-français. La seconde a été réalisée par l'équipe de la revue Liberté en 1977. Le livre d'A. Choquette a connu une réédition aux Presses laurentiennes en 1976, ce qui permet d'évaluer l'actualité des problèmes soulevés, et chacun connaît l'importance de la revue Liberté dans l'évolution de la littérature québécoise depuis sa création en 1960 et l'enquête de 1977, en passant par un autre numéro spécial qui demande u où en sont les littératures nationales ". 


\section{1) La notlon de littérature durant l'entre-deux-guerres au Québec: un manque}

En 1931, Mgr Camille Roy déduisait la vitalité de la littérature canadienne-française de la multiplication des voix qui s'y intéressaient, soit pour la louer, soit pour la ridiculiser:

"La critique littéraire a tout récemment, chez nous, multiplié ses cuvres. C'est un signe de plus grande vitalité intellectuelle. Huit volumes de critique ont paru depuis 1929, dont quatre en 1931: En feuilletant nos écrivains, par Séraphin Marion, Carquois, par Albert Pelletier, Paragraphes, par Alfred Desrochers, et Gloses critiques, par Louis Dantin. Pour le champ encore relativement pauvre de nos lettres, c'est une belle floraison.

Et tout en continuant à filer sa métaphore agricole (floraison, épis, grain, gerbe, chardon, récolte), le moissonneur termine son paragraphe par cette phrase lourde de sens sur le róle de la critique littéraire: "En littérature, comme aux champs, il faut savoir trier. ${ }^{2}$ La critique se définit donc par la nécessité du choix, l'attitude sélective élitaire. La critique s'ìmpose par un "savoir", celui de sélectionner et de classifier des textes selon des critères ou des "lois du goût et de l'esprit" (non définies) "qui se fondent ellesmêmes sur le tempérament des races». Quant à la production littéraire ellemême, en une formule négative et exclusive, Mgr Roy affirme: " La littérature, qui n'est que l'efflorescence de la vie intellectuelle... " Cette valorisation est liée chez Roy à des "lois psychologiques" (françaises) desquelles on ne peut se défaire, "plus fortes que tous les systèmes arbitraires", entendons politiques et économiques, qui pourraient tenter d'en expliciter l'enjeu. C'est ce discours idéologique que nous voudrions examiner plus à fond. Selon Mgr Roy, «la langue, la littérature et la race» définissent notre aidentité ethnique». Quels rapports ces éléments culturels entretiennent-ils avec l'ensemble de la structure sociale qui les rend possibles?

On admet aujourd'hui sans trop de difficulté que les contraintes par lesquelles le système de la production littéraire limite la liberté de l'écrivain sont d'origine socio-culturelle. Sans entrer dans les détails d'une dialectique globale du hasard (au niveau de la série diachronique littéraire) et de la nécessité (au niveau de l'extra-littéraire et surtout des rapports que la série littéraire entretient avec l'ensemble des séries culturelles et la société tout entière), retenons que l'étude immanente des textes isolés permet de comprendre ces textes mais elle ne permet pas d'expliquer le pourquoi de leur survie; pour ce faire, le généticien doit faire appel à la structure sociale englobante. L'histoire et la société sont alors considérées comme immanentes à la structure du texte littéraire, et non plus comme de simples éléments d'influences extérieures à la production littéraire. Bref, parole et écriture ne sont pas autonomes à l'égard de l'idéologie et du culte.

Puisque le contexte social est immanent à l'évolution littéraire - celleci devenant incompréhensible si elle se trouve dégagée des structures 
sociales - les problèmes esthétiques doivent donc être appréhendés comme des problèmes sociaux, n'en déplaise aux tenants idéalistes de l'art pour l'art ou de l'analyse immanente (savante) des textes.

II est vrai cependant que la théorie marxiste de la littérature tend à réduire la question du comment est écrit tel texte (son écriture) à la question socio-historique et génétique: pourquoi ce texte a-t-il été produit dans telle situation sociale donnée? Cependant, l'intention fondamentale d'un texte littéraire particulier ne saurait être saisie qu'à l'intérieur de l'évolution littéraire. Comme le remarquait Jan Mukarovsky: "Même l'artiste tient compte, en produisant une cuvre, du système normatif enraciné dans sa conscience $[. .$.$] : en cela il ne se distingue nullement des autres membres de$ la société. " Prêtre ou guerrier, pour reprendre le couple mythique d'Abellio, l'un ou l'autre définissent des fonctions sociales différentes, mais ce serait se leurrer de croire que l'activité de l'un serait plus sociale tandis que l'autre plus contemplative ${ }^{3}$. D'où il apparaît indispensable de reconnaître, sur le plan textuel, la coexistence d'éléments linguistiques et socio-économiques, le caractère normatif et social (conforme/non conforme) de la communication littéraire. Le fait littéraire ne se comprend que partiellement au niveau de la production individuelle. C'est au niveau de la consommation que son statut et sa fonction s'éclairent objectivement.

Sans trop nous attarder à examiner les conditions socio-économiques de la production littéraire des années 1920-40 au Québec par exemple, sans nous étendre non plus sur le rôle des forces collectives dans les domaines de la production et de la réception des objets symboliques, ainsi que les mutations que subissent les produits littéraires au cours de l'histoire (leurs lectures), il nous apparaît intéressant d'essayer de circonscrire d'abord la conception que se faisaient de la littérature les intellectuels québécois des années ' 30 , même si, dans l'ensemble, ils omettent d'avouer ou de reconnaître que la littérature a des origines sociales, tout en reconnaissant, paradoxalement, dans la langue, la médiation la plus importante entre la littérature et la société.

Camille Roy soutenait pour sa part les partisans du régionalisme, fidèle ainsi au groupe du Terroir qui favorisait les sujets nationaux et son idiosyncrasie terrienne, tandis que Louis Dantin de son côté, exilé à Boston, prônait une plus grande liberté dans le traitement des sujets et insistait sur la valeur esthétique des œuvres à produire.

Gaston Pilotte, dans «Victor Barbeau et le régionalisme "4 a bien montré comment Barbeau s'en prenait aux régionalistes comme L.-P. Desrosiers, $L$. Groulx, C.H. Grignon et H. Bernard5, se situant ainsi du côté de Marcel Dugas ${ }^{6}$, avec les "exotistes" du Nigog, de tendance culturelle plus centrifuge.

En 1931, le feuilletoniste Albert Pelletier ouvrait son Carquois sur cette même controverse qui touche la littérature nationale et/ou le nationalisme littéraire ${ }^{7}$. Alonzo LeBlanc, dans “ Une satire des années 1930 », a proposé un 
bel aperçu des positions d'A. Pelletier sur la littérature, la littérature nationale, la faiblesse de nos productions symboliques, la médiocrité de notre système d'enseignement, l'aliénation de nos institutions sociales en général, etc, et nous ne croyons pas utile de reprendre toutes ces observations ${ }^{8}$. En mettant l'accent sur la dimension sociologique de la pensée d'A. Pelletier, LeBlanc souhaitait contribuer à l'étude de la mentalité des intellectuels des années 1930, dans la perspective de l'article bien connu de Jacques Pelletier: « La Relève: une idéologie des années 1930 \%. A. Pelletier était un critique d'humeur, un essayiste pamphlétaire qui se débattait comme un bon diable contre les doubles contraintes de l'idéologie officielle (stérilité intellectuelle) et de la langue française mal maîtrisée (mais conforme a notre identité nationale). LeBlanc évoque avec justesse chez Pelletier, cette sorte de mépris ouvert contre l'esprit bachelier (idéologue, idéaliste, mystique), son mépris à l'égard de ce surmoi d'humanités classiques, cette part inacceptable de luimême imposée par l'éducation qu'on lui a imposée et contre laquelle il se rebiffe.

Sorte de Refus Global avant la lettre, les textes de Pelletier s'élèvent contre le classicisme officiel est ses "maîtres-garde-fous", "notre stérile rêvasserie de latins rapetissés et de dilettantes dépaysés". Une littérature nationale reste à faire; il en trouve cependant quelques ébauches dans les accents individuels et un peu frustrés de Jean-Charles Harvey, de ClaudeHenri Grignon, d'Alfred DesRochers. Chez ce dernier en particulier, il admire la virilité des hommes d'ici, le regard neuf et vivant sur le pays du Québec; il l'appelle "le plus caractéristique et le plus individuel des poètes canadiens". Prisonnier de l'esthétique dualiste, Pelletier ne retient des textes que le contenu, et contrairement à $\mathrm{L}$. Dantin qui accordait une grande importance à l'organisation formelle, il se met à la recherche de l'authenticité, du référent national, et tire ses flèches contre les "jésuites salonnards" qui méconnaissent et/ou trahissent notre identité. II faut le voir aussi faire un mauvais accueil au Glossaire du parler français au Canada préparee depuis vingt-cinq ans par la Société du parler français au Canada. II reproche aux académiciens de vouloir aseptiser notre parlure autochtone. Satire acerbe encore à propos de Mgr Roy, auteur du Manuel d'histoire de la littérature canadienne ; passant de la louange à la satire, il écrit:

Mgr Camille Roy est recteur de l'Université Laval; il fut président de la Société royale du Canada; il fait partie, à titre décoratif, de quelques autres hospices de curés en repos. C'est un personnage. C'est encore plus un homme habile. J'avoue même que Mgr Roy me paraît, en littérature, profondément machiavélique. Vous savez qu'il passa sa vie à meubler les générations d'écoliers de phrases pompeuses ou de coussinets en caoutchouc. Mais soupçonnez-vous pourquoi? Afin de rebondir indéfiniment, s'il prenait fantaisie à Jules Fournier et Olivar Asselin de faire glisser ses échasses!

J'ose à peine lever les yeux vers ce grand littérateur consacrè fétiche. Depuis qu'on me fit apprendre par cceur son manuel cabalistique d'Histoire de la littérature canadienne, il m'hypnoptise, et je ne sais que lui rendre hommage ${ }^{10}$. 
Nous avons attentivement relu surtout les Confidences d'écrivains canadiens-français qu'a recueillies Adrienne Choquette et qu'elle fit publier en 1939 aux éditions du Bien public de Trois-Rivières (réédition 1976). Nous insisterons sur le contenu des interviews, qui nous semble bien d'époque, juste avant la dernière guerre mondiale, juste avant la montée spectaculaire de l'édition québécoise durant les années de guerre, juste avant le développement du système de communication et du marché du livre, la professionnalisation du travail littéraire, la création de l'Académie canadienne-française, etc. Adrienne Choquette a interviewé trente-trois éminents écrivains (prêtres, journalistes, professeurs, etc.) à la demande de Clément Marchand, alors directeur du journal Le Mauricien. Clément Marchand voulait, à partir des propos recueillis, «tenter de délimiter le profil idéologique d'une époque de transition". (11) II projetait donc, implicitement, de faire ressortir les implications sociales (idéologiques) de la littérature et du métier d'écrivain à son époque.

Même projet par exemple dans le numéro spécial de la revue Liberté de mai-juin 1977, issu d'une enquête lancée durant l'été 1976 auprès d'écrivains de trois générations différentes et de pratiques d'écriture diverses. Ce numéro de Liberté aurait pû nous entraîner logiquement vers une réflexion plus globale sur la place de la littérature dans l'appareil scolaire, voire à quel type d'école appartient le concept pré-défini et dans quelle stratégie de reproduction il s'insère. Dans le cadre de cette recherche, nous n'avons fait que poser le problème, préférant ici nous en tenir à ceux qui parlent de littérature.

Ce type de critique journalistique soulève toutefois un problème théorique et méthodologique qu'il faut clarifier avant de nous intéresser aux questions qu'Adrienne Choquette posait à ses écrivains et aux réponses qu'elle a recueillies, puis reproduites. Le problème que nous voulons grossièrement poser concerne la place et le rôle du sujet dans la production littéraire en général:

[...] les théoriciens du Cercle linguistique de Prague se distinguent radicalement des structuralistes français, dont les théories tendent à éliminer le concept de Sujet et avec lui le problème de l'action individuelle qu'elles remplacent par la transformation structurelle. Mukarovsky réunit de manière dialectique les deux pôles dont l'opposition est sous-jacente au dynamisme de la production littéraire: l'autonomie structurelle de l'évolution [...] et l'initiative individuelle ${ }^{12}$.

II est par exemple assez peu question de technique d'écriture dans les questions d'A. Choquette, et les réponses aussi d'ailleurs. II est bien connu cependant que les intérêts sociaux s'articulent dans les changements techniques, les transgressions de normes dites esthétiques étant aussi porteuses de signification et de valeurs non esthétiques, donc partie intégrante du contenu. L'article de J.-L. Backès intitulé : "La place et le rôle de la métrique dans une théorie de la littérature", montre par exemple comment la métrique utilise à ses fins (idéologiques) propres des matériaux déjà fournis 
par la langue, cherchant avant tout à manifester son autonomie en masquant ses contradictions d'une part et en servant d'instrument de contrôle sur la production de textes d'autre part ${ }^{13}$. Pensons à ce qui distingue les sonnets d'A. DesRochers (1929) des textes de Saint-Denys Garneau (1935) et de ceux de P.-M. Lapointe (1948). Quinzes années seulement séparent les premiers des derniers textes. Chez Saint-Denys Garneau cependant, il n'y a que la forme poétique qui soit nouvelle, tandis que le Vierge incendié de Lapointe proposera une révolution et sur le plan du contenu et sur le plan de l'expression. II faudra toutefois attendre les années ' 60 pour que les poèmes automatistes passent à la littérature (c'est-à-dire dans les maisons d'édition et d'enseignement). Qu'est-ce qui motive ces changements dans la production littéraire et qu'est-ce qui détermine la reconnaissance officielle de ces transformations de forme et de contenu, après coup?

Faute de pouvoir vérifier cette prise de conscience chez les écrivains des années '30 qui parlent de leur activité d'écriture et de leur fonction reconnue comme plus ou moins sociale, faute de pouvoir faire ressortir les implications sociales de leur écriture, nous nous attarderons plutôt aux implications idéologiques du discours qu'ils ont porté sur leur écriture à eux et celle de leurs contemporains.

En d'autres termes, faute de voir comment le sujet s'inscrit dans les textes fictifs (littéraires) qu'il a produits, nous chercherons à voir comment ce sujet s'inscrit dans le discours qu'il porte sur ce qui fait de lui (et des autres) un écrivain.

Le type d'interview pratiqué par $\mathrm{A}$. Choquette oriente considérablement le discours qu'on peut porter sur l'activité littéraire. D'autre part, ce livre que nous avons choisi de traiter comme représentatif vient orienter notre propre questionnement. En cela, nous voulons respecter l'histoire, ne pas rechercher chez les Québécois des années '30 un type de réflexions qui irait de soi aujourd'hui, même si “c'est toujours d'aujourd'hui qu'on lit et l'aujourd'hui qu'on lit", remarque Meschonnic ${ }^{14}$. Les deux questions fondamentales restent: pourquoi écrire? pourquoi la littérature? Trente-cinq ans plus tard se trouvera articulé un nouveau facteur, celui de la lecture, à notre époque où on publie de plus en plus et où on lit de moins en moins, dit-on. Et c'est ce nouveau facteur qui permettra de voir en clair l'enjeu du phénomène littéraire.

Celui qui nous semble répondre avec le plus de justesse et d'à-propos aux question d'A. Choquette, c'est Clément Marchand, écrivain aujourd'hui très peu connu au Québec, mais que l'on reconnaît ici, en 1938, comme l'un des plus doués de nos écrivains, l'un de ceux "qui servent avec le plus de richesse intérieure les lettres canadiennes"; "ses premières cuvres contenaient déjà la singulière vigueur et le réalisme saisissant, unis à une grâce poétique très attachante»15.

Au départ, C. Marchand refuse la notion de "vocation" littéraire et croit hasardeuse, sinon impossible, cette manie de vouloir " démêler les influences 
mystérieuses qui déterminent chez un individu le goût de la création artistique". II faut plutôt admettre que " ce travail d'introspection ressort [...] à des impondérables"16. D'ailleurs, ni rien ni personne n'obligent aux travaux littéraires. Ce n'est donc pas la somme des lectures qui détermine chez quelqu'un le goût (ludique) d'écrire ou le besoin (travail) de faire «carrière» dans les lettres, ni l'impulsion d'un maître spirituel qui encouragerait un jeune (qui a du talent) à produire livre sur livre.

Nous reviendrons plus loin sur les "impondérables" dont parle Marchand, ce qui nous permettra de revenir sur les éléments théoriques que nous avancions au début de notre texte.

Arrêtons-nous plutôt aux concepts d'école littéraire, de système d'idées, de littèrature nationale, de carrière dans les lettres actives, de travail d'écriture et d'accomplissement de l'écrivain qui se trouve plongé dans le contexte socio-historique des années ' 30 au Québec. C'est dans ce contexte que ces notions, disséminées ici et là dans le discours de C. Marchand, prennent tout leur sens.

Quand, à longueur d'année, nous subissons l'emprise de très puissants seigneurs de la pensée française, il est bien difficile, pressés que nous sommes et travaillés d'inquiétudes, d'édifier une littérature universelle par son fond tout en demeurant particulariste dans ses cadres. Notre infériorité en ce domaine tient surtout, à mon sens, à l'indifférence, à l'impossibilité de se fixer d'un nationalisme qui ne sait qu'osciller entre deux pôles de vassalité. Elle tient encore à l'essence anglosaxonne d'institutions politiques qui, à tout moment, contrarient singulièrement nos goûts et nos façons de latins. Et que d'autres choses il faudrait dénoncer.

Il pourra, par miracle, se lever chez nous des maîtres sur le plan français, mais point sur le plan canadien, à moins de modifications radicales de nos institutions. "17

Marchand risque ici le ton de la dénonciation: le Québec est dominé économiquement et politiquement, il est colonisé parce qu'une nation (canadienne) le contrôle totalement de l'extérieur par le simple pouvoir de gouverner. Et il faudrait se défaire de cette domination politique coloniale avant de pouvoir penser se libérer de la domination impérialiste américaine. Cette situation a des conséquences graves sur les structures sociales (de classes) du pays et sur notre culture nationale. L'infériorité économique des Québécois entraîne la dépréciation de la langue et de la culture, et parler de son monde devient précaire dans sa propre langue de colonisé. La culture devient une affaire de classe. Et les nationalistes chercheront à faire croire qu'on peut régler la question nationale sans bouleverser les rapports de production capitalistes. La question nationale ne sert qu'à camoufler, dans les intérêts de la bourgeoisie nationaliste, les rapports d'exploitation internes à la société québécoise. Mais, la lutte contre l'oppression linguistique n'est pas et ne sera jamais une lutte dans la langue ou par la langue. L'idéologie nationaliste aveugle Clément Marchand ${ }^{18}$; en vérité le pouvoir linguistique 
d'une majorité (!) est directement relié à son pouvoir économique et politique, et non l'inverse.

Toutefois, Clément Marchand réussit à affirmer - ici dans une perspective nationaliste et dans des termes peu scientifiques - les rapports évidents qui s'instaurent entre la littérature et le social, entre cette pratique idéologique qu'est la littérature et cette idéologie pratique qu'est le nationalisme. Mais ce qu'il faut surtout retenir de ce type d'affirmations qui sont comme les lieux communs des lettrés de l'époque et d'aujourd'hui, c'est que l'ancrage de ce qu'on appelle une cuvre s'effectue au niveau du contenu, du c fond " comme le dit Marchand, plutôt que par le niveau de l'expression que les littéraires, s'évertuent à valoriser, avec raison d'ailleurs, mais dans une perspective esthétisante qui n'est pas défendable. C'est dire que le texte ne prend sa véritable fonction sociale que par "les systèmes d'idées "19 auxquels il peut adhérer ou auxquels il s'oppose en transgressant les contenus retenus comme conformes à une mémoire sociale donnée.

Mais le plus troublant dans tout cela, c'est que la majorité de nos intellectuels sont presque unanimes dans leur aveu d'un constat d'échec devant ce qu'on pourrait appeler une littérature québécoise. Ils peuvent certes nommer une vingtaine de noms connus qui manifestent l'existence d'une activité littéraire. Toutefois, « depuis vingt ans, il n'y a pas eu chez nous de vrais maîtres [...] de rares hommes dont les systèmes d'idées auraient pu laisser une trace profonde dans les esprits „20. Des écrivains de talent, nous en avons, mais pas de "génies", pas de ces « maîtres » qui puissent influencer et endiguer les énergies de toute une génération. Les vrais maîtres sont ailleurs; on les retrouve surtout en France, chez tous ces écrivains classiques "qui au cours des âges ont façonné une langue impérissable tout en œuvrant dans une matière universelle»21, et c'est ce classicisme-là qui devrait nous servir de modèle, sans toutefois nous laisser séduire jusqu'à la subjugation.

II semble clair que nos intellectuels des années trente, vivant à l'intérieur même des énormes parenthèses qu'ils ont tracées autour des graves problèmes soulevés par la crise économique par exemple, se désolent implicitement de l'absence d'un groupe social homogène capable de produire et de proposer un discours idéologique qui permettrait de nous définir autrement que comme des " coloniaux intellectuels de la France "22, autrement que comme de piètres imitateurs absorbés dans "le grand tout français", subjugués par le "génie français" qui a imposé sans heurt ses "normes" et ses "lois", nous empêchant ainsi de réfléchir sur nous-mêmes et de produire selon des normes et des lois qui nous seraient propres. Mais pour cela, il faudrait un cadre idéologique qui favoriserait une production autonome, il faudrait un complexe culturel que le contexte des années trente, toujours gouverné par le clergé, ne pouvait pas fournir.

[...] Seuls les génies échappent aux nécessités du travail: ils sont complets en naissant. Pour nous qui naissons dans un tel état d'indigence intellectuelle, un travail de toutes les heures est nécessaire pour 
nous créer un métier et une langue, et aussi un sens critique. Nous imitons les Français et nous les imitons bêtement.23

Il manque au Québec un groupe social bourgeois, homogène et laïc qui favoriserait un «art sincère» et libre, une littérature qui ne serait pas condamnée à l'avance par les « roquets de la pudibonderie", les “champions de l'esprit primaire", de la feinte et du mensonge; ce sont en effet les préjugés et les tabous moraux - a-politiques - qui font que notre couche épaisse de restrictions morales et de simagrées doctrinales finiront par nous étouffer définitivement, ou bien à nous réduire à n'être que de bêtes imitateurs de ce qui se fait ailleurs qu'ici. «Aux prises avec un tel état d'esprit et dans un pays où n'existe aucun sens de la solidarité, comment un écrivain aurait-il le courage de s'accomplir?...24

Nous sommes bien conscients que le phénomène littéraire ne se réduit pas à celui qui écrit et à sa production individuelle. La littérature n'est pas qu'une série plus ou moins prestigieuse de titres ou de noms rendus célèbres par un groupe social qui les a valorisés en fonction de critères plus ou moins mal définis ou plus ou moins conformes à l'objet dont ils avaient à parler, à valoriser ou à rejeter selon les besoins. Selon $\mathrm{C}$. Marchand, une littérature pourra naitre si on lui permet de naître, si ceux qui la produisent, la lisent et en parlent, arrivent à l'intégrer à un contexte socio-culturel et politicoéconomique qui la rendraient possible. Ce sont les conditions matérielles de production qui font défaut et les conditions matérielles de consommation qui sont faussées à la base. Dans l'Introduction à la critique de l'économio politique, Marx montre comment "en procurant aux produits le sujet pour lequel ils sont des produits", la consommation apparaît comme «moment de la production", pratique sociale spécifique. D'où la pauvreté ou l'inexistence d'une marchandise littéraire «personnelle» et «montrable»25.

Ecoutons la «marotte» du François Hertel de l'époque. Ce sont des lignes qu'il renierait peut-être en partie aujourd'hui, mais elles demeurent d'actualitè. Hertel était très écouté en tant que prêtre et professeur:

[...] Je m'occupe parfois d'action nationale, et je prétends que le grand devoir patriotique de l'heure est dans l'ordre de l'esprit. Entre les différentes formes de spiritualisme - le nationalisme en est une - il existe une telle fraternité, une corrélation et convertibilité si intimes que l'une ne va guère sans l'autre. D'où je conclus que le devoir national de l'heure est d'abord un approfondissement et un affermissement religieux. (... si nous avons eu si peu de maîtres de l'écriture au Québec) ne serait-ce point que les hommes les mieux doués chez nous se tournent vers l'action? Ce dont nous avons besoin désormais ce sont des maitres de contemplations, des penseurs et des poètes ${ }^{26}$.

Feignons de faire abstraction du caractère religieux de l'urgence dont il est ici question. Par-delà le refus de l'action et de l'apolitisme hautement affirmé, hélas, Hertel en appelle à la cohésion, à la stabilisation idéologique que le mouvement nationaliste québécois devrait pouvoir réaliser. Personnaliste, Hertel souhaite voir venir l'ère du réflexif. II est bien de son temps, sous 
couleur de voiler la position de classe dont est issu son discours, sous couleur de taire surtout la position fasciste que nos intellectuels de l'époque ont eu tendance à prendre sur le plan social et politique. Certains pays d'Europe leur en proposaient de beaux modèles. Quoi qu'il en soit, Hertel prêche le spiritualisme. On se croirait encore au $\mathrm{XIX}^{e}$ siècle.

En ce temps-là, en effet, l'Église canadienne rêvait depuis un siècle de l'avènement ou de la régénérescence de son passé grandiose, de son propre Paradis perdu, c'est-à-dire sa domination sur le peuple primitif confronté à la nature. Ce cadre d'une civilisation agraire d'antan feignait d'oublier qu'il s'agissait d'un peuple et d'une nature historiquement vivante, déjà au pas d'un nouveau mode de production, du capitalisme qui dissolvait " les derniers restes de l'économie fermée "27; l'Église québécoise allait s'abstraire de cette réalité, favoriser la résurgence de la société agraire par une campagne idéologique assidue que manifestent éloquemment prônes et discours, institutions publiques et privées. L'idéologie est ici entendue comme un système de croyances (ou de représentations) d'un groupe social donné et servant à justifier ou à promouvoir certaines pratiques politiques, sociales ou économiques, le plus souvent en occultant les intérêts réels que favorisent ces pratiques.

Mandements, lettres pastorales, journaux et littérature allaient donc prêter leur concours à cette idéologie agriculturiste qui idéalisait à l'excès la vie champêtre. Cette vie simple du colon ou de l'agriculteur offre en effet une division parcellaire de la société (parcelle de terre, paysan propriétaire, famille dispersée), ensemble fractionné qui ne permet "aucune division du travail, aucune utilisation des méthodes scientifiques, et par conséquent, aucune diversité de développement, aucune variété de talents, aucune richesse de rapports sociaux $» 28$.

Ce système autarcique confine les individus dans un isolement certain puisque, chaque famille se suffisant en principe à elle-même, l'échange ne se fait pas avec la société mais avec la nature. Aucune communauté d'intérêts immédiats ne peut lier ces individus épars. Dans une perspective marxiste, le système de la propriété parcellaire est donc la situation par excellence pour l'exercice d'un pouvoir car "elle crée sur toute la surface du pays l'égalité de niveau des rapports et des personnes et, par conséquent, elle offre la possibilité pour un pouvoir central d'exercer la même action sur tous les points de cette même masse» 29 .

Les individus sont condamnés à n'avoir jamais que le potentiel d'une classe. II importait aussi de maintenir le caractère primitif au peuple par l'octroi d'un dosage religieux inculquant la crainte de Dieu, le respect de l'autorité, et d'un minimum d'instruction. "Eparpillement dans l'espace" et «mentalité bornée»30 étaient les facteurs qui privaient l'agriculteur de "s'affirmer comme une puissance historique", mais c'était aussi les conditions nécessaires d'une restauration de l'Église québécoise. En niant l'industrialisation, en condamnant toutes les associations de travailleurs et toute forme de loisirs communautaires, on croyait freiner l'urbanisation, 
ralentir la montée d'une classe de travailleurs, tout en cherchant à faire cesser l'émigration des Québécois vers les villes étrangères.

Manifestant “ isolement et séparation", la campagne va donc s'opposer à la ville qui est « le fait de la concentration de la population, des instruments de production, du capital, des jouissances et des besoins " 31 .

Dans un système aussi cohérent, l'on conçoit que, le plus souvent au nom de la morale ou sous divers prétextes religieux, l'on ait censuré toutes les productions (discursives ou autres) qui avaient tendance à mettre à jour le fonctionnement du système, toute analyse qui dissertait de problèmes sociaux, de classes, de division du travail ou de répartition des tâches de direction et d'exécution. C'est ainsi que le roman comme pratique symbolique allait privilégier le genre du "terroir", comme auxiliaire de la classe qui allait le produire, en soutenant ses intérêts, en véhiculant et justifiant ses valeurs, celles d'un nationalisme mégalomane. Et l'ordre social que le roman du terroir marifeste par exemple sera peu dénoncé pour la simple raison que " la classe dominante possède exclusivement les moyens de production, de reproduction, de généralisation du sens "32. La classe dominante monopolise le langage et le soustrait à la classe dominée. Le roman ne peut donc pas représenter la réalité; il est “ représentatif de l'état idéologique, c'est-à-dire de l'image élaborée par la classe dominante et généralisée par elle pour dérober l'état de fait "32. L'envers de l'image projetée par le roman du terroir se trouvera dans La Scouine, roman qui sera alors mis à l'index, condamné comme non conforme et oublié jusqu'en 1960.

Rappelons pour ceux qui l'auraient oublié et pour ceux qui, ou bien feignaient de l'ignorer à l'époque ou bien se représentaient les conditions matérielles du Québec d'alors dans les cadres d'une représentation idéologique qui n'avait rien de commun avec la réalité économico-sociale, rappelons que la révolution industrielle au Québec date de 1860 , et non de $1920 \mathrm{comme}$ nos littérateurs ont tendance à le répéter, et même avant dans certains secteurs (chaussure, textile, bois, fabriques laitières, etc.) et que le Québec a subi la grande crise de 1929 comme un état capitaliste industrialisé. Entre 1920 et 1940, le Québec est industrialisé et urbain - Montréal compte $40 \%$ de la population - et non pas agricole comme l'idéologie physiocrate et et anti-étatiste a eu longtemps tendance à l'affirmer, même jusqu'à la "révolution tranquille" des années ' 60.

Le Québec vivait en effet dans les cadres d'un capitalisme de monopoles simples où l'oligarchie financière (fusion du capital bancaire et du capital industriel) constituait la fraction dominante de la bourgeoisie canadienne ${ }^{34}$. Le Québec représente un cas unique de sous-représentation de la majorité ethnique francophone dans la direction des "affaires" de l'État. Sa bourgeoisie était (est) en majorité étrangère (britannique, canadienne-anglaise, américaine). D'où l'impossibilité au Québec de productions symboliques d'envergure par une fraction de la petite bourgeoisie traditionnelle qui, pour contrer le capitalisme et le problème de classes sociales, proposait une idéologie qui était en retard de presque un siècle sur l'économie. 
De l'Avenir du peuple Canadien français d'Edmond de Nevers (1896) jusqu'au Précis de doctrine rurale à l'usage des Canadiens français de F. Chicoine (1948), en passant par la Vocation de la race française en Amérique de Mgr Louis-A. Paquet (1902), Orientations de l'abbé Groulx (1935) et Réflexions sur l'avenir des Canadiens français d'Edmond Turcotte (1942), tous nos idéologues officiels - à l'exception d'Errol Bouchette dont on vient de rééditer l'Indépendance économique du Canada français (La Presse, Montréal, 1977) -, tous nos idéologues, agriculturistes et contre-capitalistes d'abord, sociaux-démocrates ensuite, se sont évertués à masquer l'enjeu de luttes de classes d'une part, et à rendre fonctionnelle la lutte ethnique d'autre part, en gardant sous silence le rôle diminué et quasi nul que la bourgeoisie francophone jouait sur le plan économique. Et la production romanesque d'alors ne faisait que reproduire cette configuration idéologique. Le propre de l'idéologie étant de masquer la détermination des idées par les conditions matérielles d'existence, l'élite est contrainte de valoriser sa propre activité, même si elle ne produit que des idées, d'universaliser son projet en le proposant comme la solution aux problèmes sociaux.

On sait qu'une classe sociale se définit comme une communauté qui se distingue par le rôle et la place qu'elle tient dans la production, l'un et l'autre étant déterminés par le fait qu'elle est ou non en possession de moyens de productions. On sait également que ce sont les membres des professions libérales et ceux que l'on qualifie d'intellectuels (non salariés) - membres des classes moyennes - qui ont grosso modo favorisé l'émergence de productions symboliques dont font partie les textes littéraires. Ceux des classes moyennes ont la jouissance d'instruments de production, de création et de distribution, mais dans les limites où cette jouissance (ou propriété) les oblige à travailler pour vivre, à se définir donc, malgré eux parfois, comme des salariés, mais des salariés qui profitent de pouvoirs idéologiques importants tels les professeurs, les journalistes, les cadres intermédiaires dans la fonction publique, etc. Ces gens sont presque dépourvus de moyens importants de production, mais ils jouissent et vivent de la plus value, même s'ils ne la produisent pas. Et le désir de vouloir allier cette fraction de la petite bourgeoisie à la classe ouvrière répond aux vœux de la sociale-démocratie du Parti québécois au pouvoir depuis novembre 1976, échos lointains des partisans nationalistes qui se sont fait entendre au Québec depuis un siècle et demi ${ }^{35}$.

On pourrait en effet, au risque de voir l'histoire se répéter, comparer le texte de François Hertel et celui de Clément Marchand à un manifeste récent qui, pour plus politique qu'il n'apparaît, ne fait que reprendre sur le mode de la nécessité ce que les intellectuels des années ' 30 proposaient plutôt comme un vceu, et surtout que, depuis une vingtaine d'années tout au plus, s'affirme la nécessité d'inscrire la lutte des classes dans la lutte des langues au Québec, et réciproquement:

La réalité, c'est que nous vivons une situation politique globale, à la fois économique, sociale et culturelle, et que notre réaction à cette situation 
n'est ni folklorique ni idéologiquement sophistiquée, mais existentielle et "avancée» par son fait même, jusqu'a un certain point dans une sorte d'indifférenciation théorique (c'est nous qui soulignons). La situation du Québec a ceci d'inédit qu'elle se présente comme appelant un processus d'émancipation au sein d'une société développée.

[...]

Si les Québécois ne réussissent pas à s'affirmer en tant qu'entité majoritaire, en tant que différence, identité et altérité en Amérique du Nord et dans le monde, c'est la voie de la louisianisation. ${ }^{36 .}$

Cette "sorte d'indifférenciation théorique" a de quoi surprendre dans un texte qui nous est si contemporain, dans un discours nationaliste qui souhaite prochainement l'amorce d'un "processus d'émancipation au sein d'une société développée». Sommes-nous encore devant un projet collectif, un projet idéologique homogène? Les appels à l'action d'un abbé Groulx avaient parfois un accent plus convainquant. On l'a vu, le système économique détermine les classes sociales suivant la position des individus dans le processus de production. Ces classes tentent de s'emparer de l'institution culturelle à leur profit. Comment arrive-t-on à instituer la littérature? Et comment la fait-on fonctionner dans la société ? Qui classe les textes, pour qui, dans quel(s) but(s) ? Voilà une série de questions quauraient pu poser Adrienne Choquette à nos écrivains des années ' 30 . L'ensemble des réponses aurait sans doute manifesté chez eux non seulement une appartenance de classe mais aussi une position de classe petite bourgeoise, comme aujourd'hui d'ailleurs, classe dirigeante idéologiquement et politiquement (pouvoir élu), quand on sait très bien que la classe qui domine économiquement est ailleurs. On propose alors des stratégies d'alliance entre les différents pouvoirs de classe. C'est bien ce que disent les signataires du manifeste quand ils affirment que face à la "réalité", face à la "situation politique globale", leur "réaction [...] n'est ni folklorique ni idéologiquement sophistiquée, mais existentielle et "avancée" par son fait même”. Commentaire impuissant par excellence! On a recours à la métaphysique. La suite du texte est au conditionnel: “Si [...] à s'affirmer en tant qu'entité majoritaire [...] la louisianisation». Ce conditionnel est un souhait, non un programme, une réaction existentielle à une menace historique, non une ligne d'action.

II est malheureux de constater que le savoir moderne semble n'avoir apporté rien de positif en terme de théorie politique ${ }^{37}$. On a peut-être mal choisi notre exemple de manifeste contemporain. Il figurait pourtant dans un numéro de la revue Change consacré au Québec souverain, revue dont la notoriété progressiste (à l'affût des transformations et du "change") aurait pu nous fournir autre chose qu'une manifestation de notre piétinement idéologique collectif. Depuis les années '30, il n'apparaît pas que nos intellectuels aient proposé un "change» dans la fonction idéologique du texte (littéraire).

En tout cas, l'ensemble des propos recueillis par Adrienne Choquette nous paraissent suffisamment représentatifs de l'opinion qu'on se faisait 
durant les années '30 du phénomène littéraire, même s'il y manque les propos d'écrivains que l'on évoque (et lit) encore beaucoup aujourd'hui, tant dans les journaux, les revues et les programmes de nos institutions scolaires: Ringuet, F.-A. Savard, Saint-Denys Garneau, Jean Narrache, pour ne citer que les plus connus. Ces derniers demeurent pourtant les plus représentatifs de nos écrivains d'avant le mouvement automatiste qui se manifesta surtout après la dernière guerre mondiale. On peut regretter ces omissions simplement, en rappelant que Ringuet demeure un de nos plus grands romanciers du terroir, que Savard a incarné en Menaud le mythe séculaire et lyrique de la survivance obstinée, que Garneau fut le poète le plus marquant de son temps et le plus valorisé durant les années '50, que Jean Narrache reste le poète populiste dont les livres se vendaient le mieux à l'époque et qui fut redécouvert par les joualisants de Parti pris.

On peut donc voir dans le projet d'A. Choquette une faille symptomatique, un creux qui jette quelque lumière sur sa pratique discursive même, sur son propre mode d'intervention dans le traitement de son objet d'étude: proposer implicitement - jamais en clair - une définition du phénomène littéraire en présentant les idées d'autrui, les idées de certains parmi ceux qui pratiquent la littérature, de ceux qui tentent d'expliquer péniblement pourquoi ils écrivent, parce qu'on le leur demande, parce qu'on les fait parler, eux.

D'une manière générale, les réponses manifestent peu d'originalité, peu de marginalité, exception faite de $\mathrm{C} .-\mathrm{H}$. Grignon pour qui l'écriture est une "aventure» ou encore de Victor Barbeau pour qui "écrire est une fonction physiologique, un besoin du corps autant que de l'esprit ". Parler d'errance et de besoin du corps nous semble une marque de modernité qu'on ne pouvait pas saisir à l'époque. Dans l'ensemble, les autres écrivent pour instruire et surtout faire œuvre nationale, car c'est presque toujours cela qui importe au Québec. L'engagement nationaliste rallie la majorité des écrivains et pointe parfois la connotation religieuse que couve la notion de "vocation" (provenant de la famille ou de l'école ou de l'influence de lectures marquantes, françaises bien sûr, par l'école, mais aussi anglaises, américaines et nordeuropéennes, par affinités culturelles). C'est qu'on est toujours à la recherche de grands maitres, et en vain, surtout si on les cherche au Québec. Ce qui explique pourquoi cette carence les amène à se demander s'il existe vraiment une littérature canadienne! Non seulement ils manifestent un non-savoir, mais encore une méconnaissance de l'objet même dont ils doutent de l'existence.

\section{La notion de littérature au Québec après la révolution tranquille: une impasse}

Ce problème de la définition, de l'expansion, de la diversité et ce la fonction du phénomène littéraire a suscité une autre enquête qu'un numéro 
de la revue Liberté a reproduite sous un titre bien significatif: Divergences : la littérature québécoise par ses écrivains (mai-juin 1977). On a en effet demandé à une trentaine d'écrivains de dire comment ils voient la littérature québécoise actuelle et comment ils se situent par rapport à elle. On a compilé seize réponses très variables selon le point de vue privilégié du problème, manifestant, comme il fallait s'y attendre, des opinions "divergentes", la revue ne prenant bien sûr aucune position dans le débat, se contentant de relever quelques constantes. Tous les répondants ont fait mention d'un malaise et parfois même d'une impasse, surtout depuis 1970; depuis cette date, les écrivains prennent conscience d'une transformation du statut et du rôle social de l'écrivain au Québec. Tout se passe comme si depuis « octobre 70 " les écrivains avaient perdu cette impression de collaborer, par un type d'intervention spécifique, à un projet social collectif.

Devant les années '60, Alain Grandbois applaudissait au fait que les écrivains ne produisaient plus dans la solitude, que des lecteurs leur étaient alors fidèles, qu'une fonction sociale non définie donnait un sens à leur activité, à tel point qu'il n'était pas rare d'entendre, de la part des poètes surtout, que le poème était parole, acte, révolution, etc. C'était le bon temps de la "révolution tranquille" et de l'affirmation d'une petite bourgeoisie francophone écrivante, et lisante. Tout cela avait déjà été préparé, durant les années '50, par les collaborateurs de cité libre, dont le rôle politique important de certains, depuis dix ans, n'a pas besoin d'être démontré. D'autre part, les poètes des éditions de l'Hexagone sont devenus aujourd'hui, malgré eux souvent, les chantres classiques du pays à découvrir, à inventer, à faire naître, etc. Au même moment, nos écrivains étaient jugés dignes de figurer au programme de nos institutions d'enseignement.

L'historique des positions idéologiques de l'institution scolaire face à la "formation" des "littéraires" reste à faire. Cette carence nous oblige à nous rabattre sur le discours des littéraires eux-mêmes. Et les dépliants publicitaires des éditions de l'Hexagone sont par exemple instructifs à cet égard. Ils proposent un véritable programme de valorisation du texte poétique du Québec, “toute poésie (étant) pierre de touche d'une littérature»: participation financière des souscripteurs (avant même l'impression du recueil), accessibilité au plus grand nombre de lecteurs, diversité des tendances et valeur de continuité ( «représentatives et marquantes"!), confiance en une "action en littérature" et en l'écoute des lecteurs qui auto-financent ce qu'ils vont lire. En 1959, un dépliant publicitaire manifeste cet optimiste qui est à son comble:

Plus que des théories, c'est à partir des ceuvres, de leur situation, que se définit la réalité poétique d'un moment. [...] Nous assistons à la fin de l'aliénation du poète par la solitude stérile, la révolte à perte ou l'exil de l'intérieur. La participation de plus en plus fréquente de poètes aux luttes qui nous confrontent les a révélés à eux-mêmes et à leur réel. Chez eux, la nostalgie, la plainte, la réclusion ont cédé place à la confiance, à l'agressivité ou à l'étreinte de la possession [...] Ils savent que leur drame de poète et d'homme se joue ici, que le destin de 
l'homme peut s'enraciner dans l'expérience canadienne. Ils ne veulent plus s'aliéner dans l'ailleurs. Ils écrivent des poèmes de rupture souvent d'avec l'avant-garde reconnue ${ }^{38}$. Leur poésie est en quelque sorte une patrie [...] elle assume et nous assume [...] dans ces mille contraintes de la poésie. Voici dix ans que, au prix de silence et de durs combats intérieurs, se préparait cette éclosion. Ces poètes, sans concession, sur des modes et en des voies qui sont propres en chacun d'eux [quoi de plus opposés en effet que les textes de F. Ouellette et ceux de J.-P. Filion], parlent pour tous [...]

Unanimité donc sur la valeur culturelle et idéólogique de notre littérature, unanimité que partageront même, à un niveau différent, les écrivainsmilitants de Parti pris.

II faut relire les textes présentés à la première rencontre des poètes (septembre 1957) et réunis sous le titre La poésie et nous Hexagone, 1958). II devait être question de la place de la poésie dans notre société et de la fonction sociale du poète. Le problème était donc bien posé. II n'est pas nécessaire de résumer le contenu de ces communications, pour la simple raison que les praticiens-poètes de la littérature répondent généralement à côté, exception faite ici de G. Hénault à propos de qui nous reviendrons plus loin. Ce petit collectif sur la poésie est important parce qu'il permet d'illustrer de quelle manière on se posait la question de la pratique poétique à l'époque - et de constater par la méme occasion comment on continue toujours aujourd'hui à occulter la fonction sociale de la littérature ${ }^{39}$.

Un texte suffira, le premier, signé par M. Van Schendel sur les tendances de la poésie canadienne-française. N'insistons pas sur le préambule assez général sur la fonction de "transformation du monde" que remplit la mystique poétique moderne, et voyons comment le critique aborde la poésie qui lui était contemporaine. II avoue ne pas trop savoir comment faire et on ne peut que le comprendre: il n'a aucun recul. Cependant, il prend l'initiative de faire le partage entre les bons et les mauvais poètes:

Dans le contexte présent les tendances ne sont pas assez différenciées, et le seul partage que l'on puisse valablement faire est, à mon sens, entre les vrais et les faux poètes, entre les bons, ceux qui le sont, et les mauvais, ceux qui ne le sont pas, ceux dont on ne parlera pas. Quant à ceux dont on veut parler, un lien constant les unit: qu'ils le veuillent ou non, ils ont tous en commun une double expérience: une expérience de l'isolement, d'abord; une expérience du langage, ensuite. (c'est nous qui soulignons)

Ce «on» et ce «veut» font pour nous problème. Rien n'est précisé sur le lieu d'autorité ou de légitimation de qui parle et au nom de qui. Presque rien n'est dit non plus de ce qui justifierait de parler des «bons " poètes seulement, et de les regrouper, qu'ils le veuillent ou non (c'est très juste!), non en raison d'une fonction sociale à laquelle les textes poétiques répondraient précisément, mais en raison d'une double expérience, psychologique d'une part, à cause de l'isolement du sujet-individu-artiste, et d'autre part, esthétique, à cause de 
son travail sur la langue et plus précisément sur les "formes du langage poétique". La problématique est donc enfermée à l'intérieur du champ ou série littéraire, ce qui permet de constater des éléments de nouveauté ou de modernité dans le traitement de formes et de contenus, sans pourtant expliquer cette modernité des "bons" poètes.

On peut ne pas aimer les quelques œuvres publiées par des automatistes, mais on ne peut pas leur contester au moins un mérite: celui d'avoir engagé la poésie canadienne dans la voie des recherches.

Après un jugement de valeur inutile pour son propos (mais efficace par la réserve qu'il renferme à l'égard des automatistes), la modernité se définirait par la fécondité et la dispersion, le goût du risque, à l'aveuglette, comme “cent longs télégrammes poétiques [...] ce tas pêle-mêle d'images pas soignées". Ces jugements quelque peu péjoratifs ne sont proférés que pour mettre de l'avant des notions que nous avons déjà rencontrées à propos du classicisme impénitent de nos critiques littéraires traditionnels: en effet, après la révolte nécessaire doit maintenant faire place "la discipline du rythme", "une certaine rigueur " à opposer à "ce grand bazar des incendies et des métamorphoses"; un "inventaire" s'impose, un "tri», un "coup de balai de propreté "; la disparité des nouveaux venus, R. Giguère et J.-G. Pilon par exemple, qui "se ressemblent pourtant par leur rigueur". Tout ce qui expliquerait pourquoi ces poètes doivent être retenus à ce moment précis de l'histoire de la formation sociale qu'est le Québec d'alors, est occulté au profit de jugements de valeur soi-disants esthétiques, ne relevant que d'un "goût fermé" que, par ailleurs, ils dénoncent.

Le syntagme équivoque de "poésie sociale" hante cependant le texte de Van Schendel - comme tous les autre textes de la Poésie et nous. Van Schendel y consacre la dernière partie de son exposé. Faute de trouver au Québec une poésie " socialiste», il affirme qu'une poésie sociale « ne veut rien dire» puisque « la poésie est sociale par définition [...] par ses origines [...] par l'intermédiaire d'une forme quelconque de publication [...] qui est une rencontre sociale [...] par la pression implicite du milieu et des circonstances sur l'acte d'écrire". Compte tenu de ces réalités obligées, parler de poésie sociale, "c'est presque un pléonasme". Le critique ne manque pas ici de perspicacité ; il réussit à grouper ce qui permettrait d'amorcer une définition de ce qu'est le phénomène littéraire en général. Malheureusement, par une pirouette idéaliste, le social ne transparaît en poésie que dans un contenu explicitement social, et en ce sens il constitue un "danger», un danger pour la pureté du plus élevé des genres littéraires. Cette valorisation (idéologique) explique le pléonasme dont parle $V$ an Schendel, mais cela ne dit rien de qui ou de ce qui en décide, et pourquoi: "Pour qu'une poésie soit dite sociale, il faut qu'elle soit d'abord une poésie, et qu'elle ait ingénument des préoccupations sociales". La fonction sociale de la poésie se trouve ramenée à une question esthétique, encore une fois, à l'intérieur même du champ littéraire, concédant à ce dernier une autonomie au sein des productions culturelles, et la première place. 
Depuis 1970 environ, il semblerait que cette priorité et cette autonomie du champ littéraire soient remises en doute. En effet, un malaise et une impasse de la production littéraire vouent à nouveau nos écrivains à la solitude et au refus des messianismes idéologiques que certains critiques voudraient encore leur faire endosser. Navigation et/ou errance, la pratique littéraire exige dorénavant, affirme-t-on dans le numéro spécial de Liberté de mai-juin 1977, « un nouveau mode d'insertion, d'articulation, de la littérature dans l'ensemble de la culture", voilà le programme souhaité par la plupart de ceux qui s'y consacrent 40 .

Toutefois, l'existence d'une littérature québécoise ne fait plus de doute aujourd'hui. Tout ce qu'on peut envier aux Français d'outremer, ce serait maintenant le fait qu'ils bénéficient d'un marchè très vaste. Le nombre de titres nouveaux qui paraissent au Québec devrait pourtant être un indice de vitalité. II se publiait en 1962, vingt-six romans, trente-trois recueils de poésie et six pièces de thèâtre, chiffres qui passent à 56,73 et 16 en 1971, puis à 76 , 95 et 20 en 1975. Et que penser d'une revue annuelle comme Livres et auteurs québécois qui voit son nombre de pages gonfler d'année en année. Mais peut-on évaluer le chiffre de ventes du livre au Québec et sa résonance sociale sur le public lecteur avec les mêmes paramètres? Or, c'est précisément ce facteur de la lecture qui faisait défaut dans la définition et l'évaluation de la littérature chez nos écrivains des années ' 30 . La faculté des textes à trouver leur public, à créer ce public, c'est là aujourd'hui le nœud véritable de la problématique littéraire, lié étroitement à celui de la qualité des textes. L'enquête de la revue Liberté n'a pas été menée sous forme d'interviews, mais à partir d'un portrait-charge de notre production littéraire brossé par François Ricard:

[...] si l'on pouvait [...] calculer pour l'ensemble des œuvres publiées au Québec ces années-ci, l'impact ou le rayonnement qu'elles ont dans la vie intellectuelle de la collectivité, [...] la quantité d'idées, d'images, de références qu'elles y répandent ou éveillent, et la durée de cette influence, je me demande sérieusement si ce rayonnement, si cette mesure de résonnance sociale serait trouvée de beaucoup supérieure à ce que possédait même plus rare, la littérature québécoise d'il y a dix, quinze ou même vingt ans; ou si l'on ne verrait pas plutôt à quel point la production actuelle est inopérante, sans écho dans la collectivité et comme réduite de plus en plus à un simple bruit de surface [...], comme muselée, enfermée de plus en plus dans sa propre répétition [...], silencieuse parmi des tonnes et des tonnes de papier ${ }^{40}$.

Et de continuer sur son peu d'audience à l'étranger, ce qui accentue encore son enfermement.

Deux traits (deux plaies) de notre littérature actuelle s'expliquent en partie par là: le mouvement joualisant et "petite culture", qui est comme l'orgueil compensatoire du provincial frustré, et l'imitation maladive des modèles étrangers [...] qui est une façon de se donner la dimension internationale qu'on peut [...]. II vient un temps où d'être strictement nationaliste, une littérature ne peut plus que dépérir ${ }^{41}$. 
Tout se passe comme si notre littérature souffrait de «nationalite». Elle souffre aussi d'embonpoint parce que trop subventionnée, trop valorisée par l'État 42. Elle souffre encore de cette manie d'aller puiser à l'étranger des modèles qui ne lui conviennent pas. Enfin, elle souffre de solitude, sans impact sur le public, sans audience à l'étranger. Décidément, notre littérature se porte mal et combien elle semble difficile à définir, sans se contredire sur les éléments qui la caractériseraient, lui insuffleraient une vitalité et lui donneraient un public-lecteur.

Malgré tout, la seule approche vraiment dialectique qui convienne pour définir la littérature demeure celle qui la saisit dans sa fonction sociale. Autre idéologie, nouvelle littérature, nouveau public.

Précisément, maintenant qu'on peut affirmer l'existence et la vitalité de notre littérature, comment se fait-il que ce soit le public qui fasse défaut? C'est en effet lui qui doit accorder une valeur objective s'il le faut, à ce que produisent ces écrivains. On assiste cependant au phénomène bien connu chez les littérateurs: ils répondent à ce côté de la question parce qu'ils s'acharnent trop à définir le rôle de l'écrivain dans la société plutôt que d'insister sur la fonction que la société (ou la faction de classe correspondante) attend voir jouer à certaines de leurs productions. Nous reviendrons à la fin de notre étude sur cette commande sociale.

Tout ce que nous pouvons retenir de ces malaises et de ces impasses, de ces jeux de pendule entre le nombrilisme individuel d'un type d'écrivain (romantique) et le rôle de prophète ou de phare social (non moins romantique) d'un autre type d'écrivain, c'est qu'ils dialectisent autrement le rapport et l'intervention sociale de leur production, cette dernière étant quasi incompréhensible en elle-même, dans son autonomie d'objet. Pour Yvon Rivard, "l'écriture (est) un engagement d'autant plus exigeant qu'injustifiable». Et plus loin, confondant écriture et littérature (cette dernière étant de l'écriture institutionnalisée), "la littérature, qu'on le veuille ou non, sera toujours œuvre de prêtre et non de guerrier ".43

Gilles Marcotte affirme sans ménagement, parce qu'il voit clair au sein de l'institution: "la littérature est une industrie" et le malaise qu'on éprouve actuellement est lié à celui de “l'institution littéraire», de la machine à faire des livres, à les vendre, à les faire lire et à en parler. Que la production actuelle soit pléthorique ou décevante, cela n'enlève rien au fait que la situation de malaise dans laquelle on se trouve est normale. Après m'avoir fait sursauter, G. Marcotte m'a permis de lire enfin un propos intelligent sur le phénomène littéraire et la configuration idéologique qui lui permet d'exister avec tel ou tel type de conformité:

[...] l'ensemble de l'institution littéraire a glissé hors de la configuration idéologique qui l'englobait au cours des années soixante. Dans une telle configuration, la littérature, ou l'idée de littérature, précède en quelque sorte les œuvres. II s'agit moins de se donner des produits, des livres, qu'une industrie. D'un côté la SGF, de l'autre la CSL: la nation s'équipe. Les poèmes, les romans ne reçoivent leur pleine légitimité que 
de leur appartenance à la littérature nationale. [...] La littérature fait le pays, et le pays fait la littérature: la réciprocité est parfaite et il n'est guère d'écrivain (ou de lecteur), à cette époque, qui ne soit convaincu de la concordance entre le projet littéraire, le projet social et le projet national.

Je parle de cette configuration idéologique au présent, parce qu'elle n'est pas disparue, loin de là. [...] On peut même penser que c'est, précisément, la persistance d'une telle idéologie, devenue nostalgique, qui nous rend aujourd'hui si malheureux, si pessimistes quant à la situation présente à l'avenir immédiat des lettres québécoises. Car les choses ont changé. L'œuvre d'un Paul Chamberland nous montre presque toutes les étapes de la transformation $[\ldots]^{44}$.

Tout le texte de Marcotte est à lire; il va même jusqu'à avouer que les formes consacrées de la culture d'élite sont en butte à une étrange méfiance qui se répercute même dans les documents officiels du ministère. Et ailleurs au Canada, certains programmes gouvernementaux visent à privilégier des projets ou des entreprises qui relèvent d'une notion plus élargie, plus populaire, de la culture, plus près de la vie quotidienne d'une communauté. C'est bien la configuration idéologique qui permet de mesurer le degré de littérarité d'un texte littéraire, et non certaines marques formelles qui manifestent son appartenance à tel ou tel genre littéraire, comme la critique structuraliste a tendance à le faire croire. L'appartenance d'un texte à la littérature ne provient pas de ses qualités esthétiques d'abord, comme le croyait Van Schendel.

Entre l'idéologie de l'appartenance des années '60 et l'idéologie de la déconstruction des années '70, du démembrement, entre l'idéologie de Liberté et celle de Chroniques, précise Marcotte, la littérature change de fonction, tout comme les discours portés sur elle ou contre elle, la littérature change tant au niveau de l'expression qu'à celui du contenu. Les jugements de valeur ont par conséquent un caractère idéologique certain. La vieille complicité entre des écrivains définisseurs de situations (représentations idéologiques de nous-mêmes à l'usage de la société) et la direction effective de la conscience nationale, est aujourd'hui sclérosée, remarque Réjean Beaudoin:

Les écrivains, qui avaient doublé l'ancienne élite cléricale, après 1960 , dans la tâche de fournir ses thèmes et ses symboles à la pensée collective, les écrivains viennent d'être à leur tour doublés par une nouvelle élite de politiques (il ne faut surtout pas dire: de politiciens) dans cette même tâche de réflexion commune. (Mais) cette nouvelle élite s'est elle-même nourrie des images littéraires élaborées dans l'enthousiasme envahissant de la révolution tranquille, ce qui contribue à rendre d'autant plus étonnante la piètre valeur de la production littéraire actuelle.

\section{$[\ldots]$}

Mon impression est que l'arrivée des hommes de pouvoir oblige à une redéfinition des créateurs qui avaient insensiblement assumé, depuis 
1960, une part importante du leadership de la nation, laissée sans répondant authentique de ses options profondes, sur la scène politique: c'est cette situation qu'a modifiée radicalement la date du 15 novembre 1976 et c'est l'appréhension confuse de cette modification latente dont témoigne la vacuité relative de la production récente. [...] une nécessaire et tardive révision du rôle de l'écrivain, jusqu'ici obligé d'assumer une sorte de paternité morale qui ne lui revenait que par le défaut d'une véritable autorité politique qui soit en prise directe du dynamisme de la communauté. [...] les écrivains étaient appelés à redevenir écrivains sans plus, sans la caution de la question nationale, plus justement prise en charge par l'instance politique. [...] II faut bien comprendre l'importance du renversement que l'avènement historique du pays opère dans la littérature $[\ldots]^{45}$.

Le projet national de notre littérature semble bien devoir être mis entre parenthèses, provisoirement peut-être, l'histoire ayant tendance à se répéter. Quoi qu'il en soit, il demeure évident que la littérature ne se fabrique pas par le seul mérite ou génie de certains individus qui laisseraient en passant des textes qui s'imposeraient par le seul fait d'avoir été produits. Elle est le fruit d'un enjeu. Certains textes, comme toute production symbolique d'ailleurs, une fois légitimés et valorisés en fonction d'une conjoncture idéologique, sont faits littéraires et en appellent d'autres qui répondent au même système de représentation.

La nouvelle petite bourgeoisie, d'abord prégnante et enfin régnante au Québec, est en effet celle qui évalue et classe certains écrits comme littéraires ou non. L'exclusion de la petite bourgeoisie traditionnelle et de la paysannerie (autour de Duplessis et dans l'Eglise) du front idéologique et du champ intellectuel, a permis à la nouvelle petite bourgeoisie (urbaine) de noyauter les appareils idéologiques d'état. Elle contrôle aujourd'hui l'Appareil d'État, disposant de l'appareil répressif et maîtrisant la majorité des appareils idéologiques. Elle ne contrôle pas le Pouvoir d'État; mais grâce à son emprise sur l'Appareil d'État, elle représente la moyenne bourgeoisie québécoise qui dirige vraiment.

Alors que le politique revient à la moyenne bourgeoisie québécoise (qui dirige), que l'économique revient à la grande bourgeoisie canadienne (qui domine), l'idéologique se trouve entre les mains de la nouvelle petite bourgeoisie gouvernante québécoise. L'idéologie dominante (politiquement et économiquement fédéraliste et impérialiste) est celle de (la classe dominante) la grande bourgeoisie canadienne; l'idéologie dirigeante (politiquement sociale-démocrate) est celle de (la classe dirigeante du Québec) la moyenne bourgeoisie néo-capitaliste; l'idéologie prégnante et régnante (nationaliste “de gauche») est celle de la nouvelle petite bourgeoisie. Toutefois, ce nationalisme est aussi partagé par la moyenne bourgeoisie québécoise qui l'oppose au nationalisme fédéral canadien. Alors, la socialedémocratie n'est qu'un compromis fait à la classe-appui, le prolétariat. Pendant combien de temps encore? 
Pour le moment, la nouvelle petite bourgeoisie contrôle la production et la consommation littéraires, la littérature qui la représente. Le prolétariat en est toujours presqu'absent et elle n'a jamais été aussi illisible, aussi scriptocentriste, aussi universitaire, comme si la fonction sociale de la littérature était de la réduire à de l'écriture, et non plus l'inverse. La fonction sociale de la littérature n'est plus de reproduire le sens (l'idéologie) mais de le produire, la littérature étant comme repoussée du champ idéologique et restreinte au champ culturel (élitiste). On enseigne aujourd'hui une super-littérature. L'École, patronnée par un ministère de l'Éducation, impose ses modèles tandis que la Presse véhicule à la fois l'idéologie la plus populaire de la culture et la plus élitiste de la littérature, accentuant le fossé entre les deux.

La petite bourgeoisie consacre donc des textes qui affirment son savoir, ce dernier lui présupposant ainsi un certain pouvoir, mais fictif : c'est pourquoi sa littérature a une action si restreinte pour reprendre une expression bien connue de Mallarmé. La littérature devient écriture, production de sens; l'écriture se reproduit elle-même, enfermée, marginalisée et, faute de transformer l'idéologie qui la transforme en littérature, l'écriture se transforme elle-même, devient littérature, se fait inaccessible, se croit au-dessus des classes, et ceux qui la pratiquent la valorisent comme tel, comme métatexte...

Il suffit donc de comparer les différents corpus littéraires des diverses époques pour se convaincre de la définition de la littérature par la fonction sociale que lui attribue une formation sociale donnée. Le savoir théorique domine aujourd'hui la pratique littéraire, mais ce savoir est déterminé par le pouvoir qui a les moyens de garantir la reproduction d'un type de textes. II ne faut donc pas trop sous-estimer le rôle de la littérature qui, si elle est un effet de l'idéologie, a aussi son autonomie et peut avoir un effet récursif sur l'idéologie et par conséquent sur la sociétè. Les poètes de l'Hexagone et plus tard les écrivains de Parti pris ont travaillé à la marche de la révolution tranquille au Québec. La littérature n'est jamais gratuite ${ }^{46}$.

Devant cette nécessité de renouveler sans cesse le lieu et l'enjeu même de la lecture, nous pourrions laisser le mot de la fin au poète Pierre Nepveu: «si la poésie n'est pas un besoin pour celui qui l'écrit, comment pourrait-elle le devenir pour les lecteurs ? "47 II semble y avoir ici confusion entre le besoin, le goût et l'intérêt social, de la pratique littéraire. Nous préférons plutôt laisser le dernier mot à Jean-Marcel Léard, linguiste et sémioticien, qui travaille depuis quelques années à clarifier les problèmes touchant la science littéraire du texte (et non la science du texte littéraire, toutes les sciences pouvant en parler):

Puisque les critères de valeur sont externes et varient, que la fonction sociale des textes change avec l'idéologie, on comprend pourquoi les textes entrent et sortent de la littérature. Les traits pertinents sont variables, la littérarité est relative et inscrite dans la société et dans l'histoire. La littérature n'est pas une liste de chefs-d'œuvre éternels, un corpus stable d'œuvres ayant un fonctionnement, 
une organisation particulière. Au contraire, tous les textes obéissent aux mêmes lois gènérales de fonctionnement, aux mêmes systèmes sémiotiques (linguistique et narratif) ${ }^{48}$.

\section{Notion, catégorie et concept de la littérature}

Pour revenir à notre propos du début, la littérature se définit donc par sa seule fonction sociale. Ignorer cette dernière, c'est s'interdire de saisir le phénomène littéraire dans son ensemble. Le texte littéraire ne doit donc sa spécificité qu'en tant qu'objet social: pour devenir littéraire, il faut que le texte soit accepté par la communauté dans laquelle il fonctionne. La littérature étant de l'écriture transformée par de l'idéologie, le corpus ou l'anthologie littéraire est déterminée par des critères de sélection variables imposés par un groupe social élitiste qui fait vivre le phénomène littéraire et qui permet à un texte de passer ou non à la littérature selon qu'il est conforme ou non à la fonction qu'il doit remplir dans une configuration idéologique donnée. II faut donc toute une infrastructure capable de l'intégrer: dans une société qui fait de la littérature un objet d'enseignement, la sélection des éditeurs et des critiques se trouve relayée par celle des chercheurs, des manuels scolaires et finalement des enseignants. Au Québec, il faudra attendre les années ' 60 avant que l'appareil scolaire rende valide l'enseignement de la littérature québécoise jugée jusqu'alors plutôt mineure comparativement à la littérature française européenne.

L'enjeu capital du discours sur la littérature se manifeste, tout compte fait, lors de l'intervention du facteur de l'édition car, par le manuel notamment, les revues et les textes critiques (universitaires), l'édition se situe au carrefour où s'interpénètrent pratique commerciale et commerce intellectuel.

Dans un article remarquable à bien des égards, Pierre Kuentz a fait apparaître l'enjeu du discours que l'on porte sur les textes dits littéraires dans le système social qui le rend possible et efficace (nécessaire). Kuentz a surtout axé son analyse en remettant en question le postulat que présuppose les manuels traditionnels de littérature, celui de la clôture fictive de l'univers textuel, ce dernier étant posé comme une totalité homogène. Le manuel-type se présente en effet comme un nceud dans un réseau de relations, le carrefour de deux chaînes hétérogènes, celle de la série de l'enseignement et celle de la série de l'édition. La notion de littérature n'apparaît plus désormais qu'un type particulier d'appropriation des cuvres d'art, lié aux conceptions de la bourgeoisie et aux instances par lesquelles le système s'auto-reproduit, notamment à l'école.

Les liens que nous avons constamment soulignés entre littérature et discours sur la littérature [...] ne sont pas des liens circonstanciels. II ne suffit donc pas de dire, avec R. Barthes: "La littérature est ce qui s'enseigne. Un point c'est tout." La littérature en fait ne s'enseigne comme telle que depuis la révolution bourgeoise et la question qui se trouve ainsi posée est celle-ci : pourquoi cet enseignement apparem- 
ment si peu rentable a-t-il été développé par une classe soucieuse plus que toute autre de rentabilité ? [...]

Ce que devait produire cet enseignement [...] c'étaient les rapports de production correspondant à cette étape nouvelle du développement des forces de production. Aussi ne s'agit-il pas de le situer relativement à des rapports, dont il ne serait que le reflet "spirituel ", mais bien de le situer dans ces rapports, dont il est un des supports. Sous couleur d'enseigner les belles-lettres ce que l'on enseigne en réalité [...] c'est un comportement social. [...]

Mais en fait, ce privilège apparent de l'auteur n'est que l'envers du statut que l'on veut imposer au lecteur. Le principe des manceuvres de l'idéologie réside dans la fausse scansion, celle qui ne pose l'endroit que pour poser dans l'ombre son envers. II semble que l'on s'efforce de promouvoir la consommation de la production littéraire; il s'agit, en réalité, d'assurer la production du consommateur. Le culte de l'auteur sert à déterminer le modèle du lecteur: on ne lit plus les "classiques", sitôt quittée la classe, mais on a appris un certain type de lecture [...] privée ${ }^{49}$.

Ceux qui se sont amusés à interroger leur entourage sur leurs auteurs préférés, dans le but très souvent de dénoncer la série d'ambiguités qui a toujours accompagné la notion de littérature, ceux-là ont bien vite constaté que la connaissance de la littérature provient généralement beaucoup moins de la lecture que du ouï-dire social, et que dans l'étude du phénomène littéraire - écriture, lecture et critique - il est nécessaire de faire intervenir des mécanismes sociaux "extérieurs" à la littérature. Lorsqu'on essaie de définir cette dernière, on doit sortir de la série littéraire proprement dite. En se posant les "bonnes questions", à savoir qui écrit (problème de la genèse des textes et du sujet), pour qui (leurs lecteurs) et pourquoi (leur fonction esthétique et/ou leur fonction sociale, et les critères de sélection et de valorisation qui déterminent la constitution du corpus littéraire, instable à travers l'histoire), la littérature se trouve alors incorporée à l'histoire des productions symboliques en dehors de laquelle elle n'a plus de signification, c'est-à-dire qu'elle devient incompréhensible.

Les premiers travaux d'Escarpit ont été conditionnés, de son propre aveu, par une révolte contre l'institution littéraire. Plus autobiographiques qu'épistémologiques, ses recherches tournent poutant autour de questions fondamentales: se demander "que peut la littérature ?" est déjà une attitude plus scientifique que se demander "qu'est-ce que la littérature?", mais il serait mieux encore de se demander «que pouvons-nous faire de la littérature? "50. II nous a semblé plutôt que définir l'objet de la littérature était autre chose que de définir le phénomène littéraire dans son ensemble. Quant à savoir ce que l'on peut faire aujourd'hui de la littérature, il faut d'abord bien voir où elle se situe dans le champ de la connaissance (scientifique) actuelle, voir à qui elle s'adresse et qui elle sert. Tout le reste relève d'une activité sociale que l'on valorise ou pas selon la place qu'on accorde à cette activité dans l'institution culturelle de notre formation sociale. 
Depuis toujours, les adeptes de la littérature, les critiques comme on les appelle en général, qu'ils soient de l'ancienne critique (éthique, interprétative, tautologique) ou de la nouvelle critique (descriptive, formaliste, esthétique, mais tout aussi idéaliste à propos de la "littérarité" des textes... déjà retenus comme littéraires), les critiques ont toujours justifié leur discours et leur fonction institutionnelle en se demandant: comment parler à la littérature? De plus en plus, les études littéraires sont envahies par la théorie qui met à jour les mécanismes plus idéologiques que scientifiques de la pratique critique. La théorie se demande: comment parler de la littérature et non de quelques textes isolés ici et là.

Théorie du texte et de la signification; théorie de l'œuvre et du sens; théorie du signifiant; théorie de la signifiance; théorie de l'écrit et de l'écriture; théorie de la scripture, en ajoutant les théories du signe, du langage, de la fiction, du sujet, de l'histoire... tout cela pour souhaiter mettre en forme une théorie et une science de la littérature qui traiterait de la question littéraire dans toutes ses implications : linguistique, psychanalytique, sociologique, etc. Quel programme! Et quelle macédoine aussi! Confusion constante entre écriture et littérature, confusion qui élève l'une et/ou l'autre au rang du mythe et perpétue le mystère de la pratique littéraire.

Jusqu'à la fin du XIX $X^{e}$ siècle, la littérature était pensée par ses seuls praticiens. Devant l'essor considérable de l'édition, la bourgeoisie a eu recours aux critiques littéraires afin que ceux-ci instituent la valeur littéraire. Depuis 1960 environ, depuis que la littérature est pensée par des théoriciens qui en étudient la pratique, cette notion de littérature s'est vue remplacée par une catégorie, la littérarité; et parallèlement à cela, un travail de démystification de la littérature par des praticiens "avant-gardistes" mettait à la mode la notion d'écriture et celle de texte, réussissant à démontrer que la littérarité n'est rien d'autre que l'adéquation (idéologique) d'une écriture à la fonction sociale de la littérature d'une société. C'est pourquoi les critères de littérarité varient et pourquoi les corpus dits littéraires changent. Lorsque la littérature traditionnelle peut être qualifiée d'écriture de l'idéologie, la littérature moderne apparaît aujourd'hui comme idéologie de l'écriture. Ainsi l'étude traditionnelle a tendance à réduire la littérature à de l'idéologie et l'étude moderne à de l'écriture. Les deux approches risquent de passer à côtè de la littérature, la refoulant en quelque sorte, faute d'une théorie matérialiste. Il faut donc admettre la fonction sociale de la littérature, propre à chaque époque et à chaque société.

La catégorie qu'est la littérarité et la notion de littérature (et/ou écriture) demeurent les obstacles épistémologiques majeurs à la constitution d'une véritable théorie qui ferait de la littérature un objet de connaissance, qui en délimiterait le concept théorique fondamental. L'hypothèse théorique que nous avançons, à savoir que la littérature se définit par sa fonction sociale, permet de passer de la théorie littéraire à la théorie de la littérature, le concept de fonction sociale de celle-ci étant l'équivalent épistémologique du concept de texte pour celle-là (qui réduit la littérature à une certaine pratique de l'écriture). 
Pour connaître la littérature (la décrire, la comprendre et l'expliquer) il ne suffit pas de décrire le fonctionnement des œuvres littéraires, même traité en fonction d'une typologie des discours (littéraires); il faut aussi comprendre le phénomène littéraire dans son ensemble. Il faut éviter ou bien de le réduire à de l'écriture ou bien à de l'idéologie, car elle est pratique idéologique (sociale) avant d'être idéologie pratique (littéraire et nationale) 51 . II faut donc faire appel à des sciences externes comme la psychanalyse (le sujet dans le discours) et la sociologie (l'idéologie et l'histoire). La sociologie étudie les conditions de production et de consommation des textes dans une formation sociale donnée. Mais, si ces sciences peuvent comprendre le phénomène littéraire, peuvent-elles expliquer les textes par le phénomène? II apparaît indispensable d'analyser la fonction sociale (historique) de la littérature pour l'expliquer, non seulement dans et par le contexte mais dans et par le texte même qui se présente comme la solution (imaginaire) de la contradiction de la littérature entre l'écriture et l'idéologie, et du texte entre géno-texte et phéno-texte, et du discours entre langue et langage. Enfin, il faut faire appel au matérialisme qui pose les bonnes questions: pour qui et pourquoi?

\section{Robert GIROUX Université de Sherbrooke}

1. Robert Escarpit, Le littéraire et le social, Champs, Flammarion, Paris, 1970, p. 12 et 13 .

2. Mgr Camille Roy, "Critique et littérature nationale" in Regards sur les lettres, 1931.

3. En ce sens, le romancier Yves Beauchemin a tort d'écrire: «Comme citoyen, j'essaie d'agir. Mais comme écrivain, je suis forcé d'attendre. (...) Pour moi, un écrivain, méme dans la fiction la plus débridée, reste avant tout un contemplatif, la conscience de sa nation. (...) je ne conçois pas d'autre façon d'écrire que de mettre ses tripes sur la table." (Revue Liberté, no III, Montréal, mai-juin 1977. p. 19).

4. Études françaises, VII, fév. 1971, p. 23-47

5. Harry Bernard, Essais critiques, Montréal, Ed. A.C.F., 1929.

6. Marcel Dugas, Littérature canadienne-française: Aperçus, Paris, Firmin-Didot, 1929.

7. Anthologie de textes d'Albert Pelletier préparée par Lucien Parizeau, Ecrits du Canada français, Montréal, H.M.H., no 34, 1972, Cf. Carquois, Montréal, Éd. A.C.F., 1931

8. Alonzo LeBlanc, "L'œurre d'A. Pelletier: une satire sociale des années 1930 ", Voix et images du pays VI, Montréal, P.U.Q., 1973.

9. Voix et images du pays V, Montréal, P.U.Q., 1972.

10. Cité par Alonzo LeBlanc, art.cit., p. 45.

11. Adrienne Choquette, Confidences d'écrivains canadiens-français, Les Presses Laurentiennes, Québec, 1976, p. 5.

12. "De la structure textuelle à la structure sociale" de Pierre Zima, Revue d'esthétique, 2/3, Coll. 10/18, 1976, repris dans Pour une sociologie du texte littéraire, 1\%,18, 1978, no 1238. Mais ce qui caractérisait le travail des Formalistes russes, c'est le décalage entre la richesse de leur technologie et de leur méthodologie littéraires 
et l'indigence de leur théorie. D'où cette tendance à prendre pour une théorie cette technique et cette méthode de lecture et d'analyse. D'où leurs contradictions, notamment le rejet du psychologisme inhérent à la notion de personnage qui s'accompagne de sa réinsertion dans la notion d'auteur. Le principe d'immanence qu'ils prônaient entraîne la conception de structures préexistantes à « dénicher " dans le texte. D'où l'accent porté plus sur le fonctionnement des textes que sur leur production. II reste qu'ils ont permis de limiter la notion mythifiante de création, limiter la portée de la notion de représentation en refusant de conférer aux œuvres une fonction purement référentielle. Lire "La production littéraire: métaphore, concept ou champ problématique? ? de Nicole Gueunier, Littérature, no 14, Paris, mai 1974.

13. Littérature, no 14 , mai 1974, p. 19-35. Selon Backès, il serait plus efficace de tenter de circonscrire ce qu'est l'idéologie littéraire plutôt que de se demander ce qu'est la littérature. La théorie de la littérature aurait ainsi une raison d'être comme branche spécifique de la théorie des idéologies. Ensuite, l'objet d'une science de la littérature pourrait être construit comme l'ensemble des textes organisés par une idéologie littéraire.

14. Henri Meschonnic, Pour la poétique II, Paris, Gallimard, 1973, p. 37.

15. A. Choquette, op. cit, p. 159.

16. A. Choquette, op. cit., p. 160.

17. A. Choquette, op. cit, p. 164.

18. Même aveuglément idéologique chez Mgr Camille Roy. Même dénonciation colonialiste toutefois. Mgr Roy termine en effet son article en rappelant les paroles d'un écrivain et critique Canadien anglais, M. Wilson MacDonald, qui dénonçait le colonialisme comme obstacle à la création d'une puissante littérature chez nous. Et de conclure : "Colonialisme ou dépendance britannique: cela maintient chez nous des habitudes d'esprit où le manque d'initiative nationale, le manque de larges horizons politiques, l'absence de problèmes internationaux, joints à la timidité ou à l'insouciance qu'engendre toujours une tutelle quelconque, dispensent volontiers de l'effort ou le paralysent. " D'où notre solitude, notre sujétion, notre banalité...

19. A. Choquette, op. cit., p. 163.

20. A. Choquette, op. cit., p. 163.

21. A. Choquette, op. cit., p. 162.

22. A. Choquette, op. cit., p. 163.

23. A. Choquette, op. cit., p. 165.

24. A. Choquette, op. cit., p. 166.

25. A. Choquette, op. cit., p. 165.

26. A. Choquette, op. cit., p. 143.

27. Kostas Papaiouannou, Les Marxistes, Paris, Éd. J'ai lu, 1965, p. 137.

28. Ibid., p. 216.

29. Ibid., p. 217.

30. Ibid., p. 82.

31. Ibid., p. 104.

32. Paul Grivel, Production de l'intérêt romanesque, La Haye, Mouton, 1973, p. 335.

33. Ibid., p. 226.

34. La production agricole est supplantée par les industries des pâtes et papiers, de la construction de wagons de chemin de fer et d'accessoires, des textiles, etc. Lire les 3 articles consacrés à «notre héritage des années 30 » de Voix et images, vol. III, no 1 , sept. 1977.

35. Nous proposons à ce sujet la lecture de deux excellentes thèses de doctorat de I'Université de Sherbrooke (Québec), soutenues en 1978: "Espace de l'homme, espace de la femme dans le roman du terroir canadien-français" par Janine Boyard-Frot, et « Essai de théorie littéraire: le roman canadien-français du XIXe siècle" par Pierre Sallenave. 
36. «Réflexion à quatre voix sur l'émergence d'un pouvoir québécois", in Change, nos 30-31, Seghers/Laffont, Paris, mars 1977, consacré au “Québec souverain», p. 6 et 9 .

37. "Selon la théorie du matérialisme historique, explique Jean-Renaud Seba, la connaissance d'une idéologie ne passe pas par sa formalisation - que celle-ci soit énoncée en termes logiques (les axiomes).ou en termes linguistiques (les codes) - mais bien, au contraire, par l'analyse de son intervention dans la pratique sociale. Une idéologie en effet n'est pas un ensemble d'idées (... mais) un ensemble qui intervient dans un complexe de pratiques contradictoires. Le propre de cette intervention, c'est qu'elle est une des conditions d'existence des pratiques où elle intervient, lesquelles, dans les sociètès de classes, renvoient toujours, en dernière instance, à la pratique d'exploitation économique et à la pratique de domination politique, c'est-à-dire en définitive à la lutte des classes. Corollaires: un ensemble d'idées sans intervention pratique n'a pas d'existence sociale; et une analyse d'idées qui ne met pas en lumière leur intervention pratique n'a pas d'existence scientifique". ("Critique des catégories de l'histoire de la littérature: téléologie et réalisme chez Lanson ", Littérature, no 16, déc. 1974.

38. Cette avant-garde reconnue semble vouloir rappeler les "automatistes" que l'internationalisme et leurs audaces formelles ont acculés à une impasse... bien sûr.

39. Nous avons tenu pour négligeable l'enquête menée par V.-L. Beaulieu dans Quand les écrivains québécois jouent le jeu! (Ed. du Jour, 1970), quoique le titre soit significatif. Ces 43 réponses au questionnaire Marcel Proust nous paraissant trop superficielles, mondaines, complaisantes et inutilement intimistes. A retenir peut-être à la question: votre occupation préférée, les réponses de R. Duguay: "Parler pour ne rien dire", et de C. Gauvreau: "Écrire».

40. "Le problème posè", Liberté, mai-juin 1977, p. 10-11.

41. Ibid., p. 12.

42. Dans "La littérature post-nationale", Réjean Beaudoin va jusqu'à affirmer: "Au pays des artisans, des gigueurs et des violoneux, on ne consulte pas le psychiatre: on demande une bourse de création!" Une partie importante de notre souveraineté culturelle se trouve gérée par des politiques fédérales. "Quelqu'un aurait-il intérêt à noyer le sens dans le déferlement de l'insensé, qu'il n'agirait pas autrement." (Liberté, mai-juin 1977, p. 25).

43. "L'épreuve du regard", Liberté, mai-juin 1977, p. 94.

44. "Les problèmes du capitaine", Liberté, mai-juin 1977, p. 82-83.

45. "La littérature post-nationale" de Réjean Beaudoin, Liberté, mai-juin 1977, p. 26 à 29.

46. Ce qui complique l'étude de la littérature québécoise, c'est que le Québec n'est pas (encore) un État; il est une société au sein de la formation sociale canadienne. Un autre problème: le nationalisme, qui est une idéologie (pratique) de classe. Au Québec, il a touché toutes les classes à travers son histoire. De plus, il existe deux nationalismes au Canada: le fédéralisme de la grande bourgeoisie canadienne et l'indépendantisme de la moyenne bourgeoisie québécoise (et d'autres provinces). On parle d'ailleurs de séparatisme, le nationalisme ne transcendant pas les classes. En fin de compte, il n'y a pas plus de nationalisme national que de littérature nationale ou de littérature universelle; il n'y a qu'une littérature de classe.

Un autre problème, la langue, les rapports entre le français et l'anglais, le francoquébécois et le français international, un autre problème de classe. Jusqu'en 1960, le champ littéraire était relié au champ linguistique, la bonne langue fondant la littérature. A partir des années ' 60 se manifeste une rupture, non entre la littérature et la langue, mais entre la littérature et le français international: l'exploitation du "joual" national devient un critère de littérarité, le théâtre envahissant alors le corpus de l'époque. Depuis 1970 apparaît une rupture entre la langue et la littérature, celle-ci utilisant l'autre davantage comme matériau que 
comme véhicule (langage), plus pour signifier que pour communiquer, un français fictif. Tandis qu'autrefois la langue se faisait littéraire, aujourd'hui, la littérature se fait jeu langagier.

47. “La poésie et quelques questions", Liberté, mai-juin 1977, p. 89.

48. «Linguistique structurale et sémiotique dans la théorie et la méthodologie de la littérature", Le journal canadien de recherche sémiotique, automne 1976.

49. Pierre Kuentz, "L'envers du texte», Littérature, no 7, oct. 1972. p. 25-26.

50. Escarpit, op. cit., p. 41.

51. Cette réserve à l'égard des études immanentes modernes n'enlèvent rien à l'intérêt des analyses que permet l'utilisation des instruments de la sémiotique par exemple. Des communications au congrés de l'A.C.F.A.S. de mai 1978 ont prouvé que le fait de pouvoir décrire le fonctionnement textuel avec tel ou tel instrument sémiotique arrive à qualifier sa plus ou moins grande modernité. Cette dernière se manifeste avec la disparition des niveaux d'intégration (phrase, histoire) et du référent, niant par là l'opposition traditionnelle prose/poésie. Mais le fait de constater le passage fondamental de l'étape sémantique (intégration et référent) à l'étape sémiotique (le texte se constituant sur des relations de signe à signe) comme critère de modernité, ce fait est décrit, mais il ne peut être expliqué par la sémiotique. 